\title{
Acupuncture Alters Expression of Insulin Signaling Related Molecules and Improves Insulin Resistance in OLETF Rats
}

\author{
Xin-Yu Huang, ${ }^{1}$ Liang Zhang, ${ }^{1}$ Jian Sun, ${ }^{2}$ Neng-Gui Xu, ${ }^{3}$ and Wei Yi ${ }^{1}$ \\ ${ }^{1}$ Clinical Medical College of Acupuncture and Rehabilitation, Guangzhou University of Traditional Chinese Medicine, \\ Guangzhou, China \\ ${ }^{2}$ Guangdong Provincial Hospital of Traditional Chinese Medicine, Guangzhou, China \\ ${ }^{3}$ Guangzhou University of Traditional Chinese Medicine, Guangzhou, China
}

Correspondence should be addressed to Neng-Gui Xu; ngxu8018@gzucm.edu.cn and Wei Yi; ywhtcm@163.com

Received 31 March 2016; Accepted 27 June 2016

Academic Editor: Giuseppe Caminiti

Copyright (C) 2016 Xin-Yu Huang et al. This is an open access article distributed under the Creative Commons Attribution License, which permits unrestricted use, distribution, and reproduction in any medium, provided the original work is properly cited.

To determine effect of acupuncture on insulin resistance in Otsuka Long-Evans Tokushima Fatty (OLETF) rats and to evaluate expression of insulin signaling components. Rats were divided into three groups: Sprague-Dawley (SD) rats, OLETF rats, and acupuncture+OLETF rats. Acupuncture was subcutaneously applied to Neiguan (PC6), Zusanli (ST36), and Sanyinjiao (SP6); in contrast, acupuncture to Shenshu (BL23) was administered perpendicularly. For Neiguan (PC6) and Zusanli (ST36), needles were connected to an electroacupuncture (EA) apparatus. Fasting blood glucose (FPG) was measured by glucose oxidase method. Plasma fasting insulin (FINS) and serum C peptide (C-P) were determined by ELISA. Protein and mRNA expressions of insulin signaling molecules were determined by Western blot and real-time RT-PCR, respectively. OLETF rats exhibit increased levels of FPG, FINS, $\mathrm{C}-\mathrm{P}$, and homeostasis model assessment-estimated insulin resistance (HOMA-IR), which were effectively decreased by acupuncture treatment. mRNA expressions of several insulin signaling related molecules IRS1, IRS2, Akt2, aPKC $\zeta$, and GLUT4 were decreased in OLETF rats compared to SD controls. Expression of these molecules was restored back to normal levels upon acupuncture administration. PI3K-p85 $\alpha$ was increased in OLETF rats; this increase was also reversed by acupuncture treatment. Acupuncture improves insulin resistance in OLETF rats, possibly via regulating expression of key insulin signaling related molecules.

\section{Introduction}

Diabetes mellitus (DM) is a significant public health issue worldwide. Methods for controlling plasma glucose levels in patients with DM include diet, exercise, and medication. While most medications are convenient and effective, they may be associated with serious side effects $[1,2]$. As such, much research has focused on identifying alternative approaches to increase insulin sensitivity in patients with DM [3-6]. Insulin resistance is the predominant factor contributing to metabolic disorders associated with type 2 diabetes mellitus (noninsulin-dependent, T2DM) [7]. Since T2DM is the most common type of diabetes, improving insulin sensitivity is an important clinical goal.

Traditional Chinese medicine for the treatment of DM includes syndrome differentiation, electroacupuncture (EA), and Chinese massage (Tui na) $[3,8-10]$. Much work has shown that EA and Tui na not only decrease plasma glucose levels but also stimulate the neuroendocrine system, the cardiovascular system, and the digestive system to either directly or indirectly regulate plasma glucose $[4,5,11]$. Acupuncture has been practiced in China for thousands of years, and, more recently, it has become a popular therapeutic option for various malignancies in a number of other countries worldwide [12]. Studies have shown that stimulating the CV-12, CV-4, and ST-36 acupoints on both sides of a rat model with a specific frequency significantly reduces plasma glucose levels [4, 13]. Stimulation of acupoints lowers plasma glucose levels to a greater extent than does stimulation of adjacent nonacupuncture points [4]. Numerous studies have demonstrated that acupuncture can correct various metabolic disorders that contribute to the development of insulin resistance, including hyperglycemia, obesity, hyperphagia, hyperlipidemia, inflammation, altered activity of the 
sympathetic nervous system, and insulin signaling defects [14]. Although acupuncture has the potential to improve pathological conditions [15], the mechanism of the potential effect on insulin resistance remains elusive.

Altered expression or signaling of the insulin signal transduction pathway is a common occurrence associated with insulin resistance. For example, decreased expression of insulin receptor substrate-1 (IRS-1) or reduced serine phosphorylation of this protein is associated with insulin resistance, and, importantly, such alterations influence downstream signaling via phosphatidylinositol-3 kinase (PI3K) [16-18]. These proteins are key molecules involved in insulin signal transduction. Binding of insulin to the insulin receptor (IR) induces autophosphorylation and subsequent tyrosine phosphorylation of docking proteins IRS-1 and IRS-2. Activation of IRS-1 and IRS-2 leads to downstream signal transmission via PI3K. PI3K phosphorylates PIP2 to generate PIP3, which then activates phosphoinositide-dependent kinase (PDK). PDK then activates both Akt (protein kinase $\mathrm{B})$ and protein kinase $\mathrm{C}(\mathrm{PKC})$, which influence expression of glucose transporter 4 (GLUT4) [19, 20]. Expression of GLUT4 is important for proper glucose metabolism, particularly in skeletal muscle where it is highly expressed. Within this tissue type, insulin regulates GLUT4 activity to stimulate glucose transport in an Akt/PKC-dependent manner $[21,22]$.

Otsuka Long-Evans Tokushima Fatty (OLETF) rats exhibit spontaneously elevated blood glucose levels, and, as such, serve as an animal model of type 2 diabetes mellitus (T2DM) [23]. Here, we examine the effect of acupuncture both on insulin resistance in OLETF rats and on the expression of insulin signaling components in rodent skeletal muscle. We find that acupuncture significantly improves insulin resistance in OLETF rats, possibly via regulating expression of key insulin signaling related molecules. These findings suggest that acupuncture may be a valuable treatment option for rats suffering from T2DM.

\section{Materials and Methods}

2.1. Animals. Healthy male Sprague-Dawley (SD) rats weighing $222.6 \pm 22.6 \mathrm{~g}$ and aged 10 weeks were purchased from the Experimental Animal Center of Guangzhou University of Chinese Medicine (China). Male Otsuka Long-Evans Tokushima Fatty (OLETF) rats (body weight: $360.0 \pm 35.6 \mathrm{~g}$ ), genetic models of hyperglycemia that were developed in the Research Laboratory of the Otsuka Pharmaceutical Company [23-25], were supplied from the Research Laboratory of Otsuka Pharmaceutical Company at Hamamatsu (Japan) at 5 weeks of age. Animals were maintained in the Experimental Animal Center of Guangzhou University of Chinese Medicine with free access to water and food for 14 weeks. Animals were housed in Plexiglas cages at a constant room temperature of $20 \pm 2^{\circ} \mathrm{C}$ in a controlled environment and received $12 \mathrm{~h}$ of artificial light per day with a relative humidity of $60 \pm 10 \%$.

Animals were ethically and humanely treated in accordance with the guiding principles for the Care and Use of

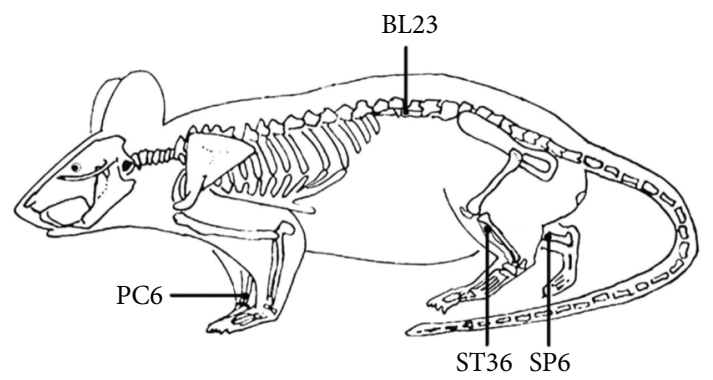

FIGURE 1: Schematic diagram indicates the four selected acupoints: Neiguan (PC6), Sanyinjiao (SP6), Shenshu (BL23), and Zusanli (ST36); these points correspond to equivalent acupoints in humans. Neiguan: located at medialis forelimbs between the radius and ulna, approximately $3 \mathrm{~mm}$ from the wrist to the elbow. Regional anatomy: between the deep flexor digitorum, where median nerve and median artery and vein travel through. Zusanli: located at the posterolateral knee of hind limbs, about $5 \mathrm{~mm}$ below the fibular head. Regional anatomy: between the $\mathrm{m}$. anterior tibialis and $\mathrm{m}$. extensor digitorum longus, where peroneal muscles, peroneal nerve, and anterior tibial artery and vein travel through. Sanyinjiao: located at the medial side of the hind leg, $10 \mathrm{~mm}$ directly above the tip of the medial malleolus. Regional anatomy: where the flexor digitorum profundus muscle, tibial nerve, and posterior tibial artery and vein travel through. Shenshu: located in the lumbar region, at the same level as the inferior border of the spinous process of the second lumbar vertebra (L2). Regional anatomy: where the fascia lumbodorsalis, $\mathrm{m}$. longissimus and $\mathrm{m}$. iliocostalis, hip lumbar artery, lumbar vein, and spinal nerve travel through.

Laboratory Animals. The study protocol was approved by the Animal Experimental Committee of Guangzhou University of Chinese Medicine.

2.2. Study Design. Rats were divided into three groups: control group (SD rats, $n=8$ ), hyperglycemic model (OLETF rats, $n=8)$, and acupuncture+OLETF group $(n=8)$. Acupuncture was applied to bilateral Neiguan (PC6), Zusanli (ST36), Sanyinjiao (SP6), and Shenshu (BL23) [26]. Sterilized, disposable, stainless steel acupuncture needles $(0.35 \times$ $33 \mathrm{~mm}$ ) were obtained from Huatuo Medical Devices Co. Ltd. in Suzhou, China. The positions of acupoints described in this study are described in Figure 1.

Operation. Neiguan (PC6), Zusanli (ST36), and Sanyinjiao (SP6) were subcutaneously probed at a depth of $8 \mathrm{~mm}$. Shenshu (BL23) was administered perpendicularly at $5 \mathrm{~mm}$. Needles used for Neiguan (PC6) and Zusanli (ST36) were connected to a G6805-2A low-frequency electronic impulse electroacupuncture apparatus (Huayi Medical Instrument Co., Ltd., Shanghai, China); this included administration of sparse-dense waves (sparse wave $2 \mathrm{~Hz}$, dense wave $50 \mathrm{~Hz}, 2 \mathrm{~s}$, resp.) for $20 \mathrm{~min}$, once per day, for a total of 21 days.

2.3. Sampling. All rats were fasted for 12 hours after the last acupuncture treatment. On the second morning, blood samples from the orbital venous sinus were collected. Samples were measured for plasma fasting blood glucose (FPG), plasma fasting insulin (FINS), and serum $\mathrm{C}$ peptide (C-P) 
TABLE 1: Primers for real-time PCR.

\begin{tabular}{|c|c|c|}
\hline Gene & Primer $\left(5^{\prime}-3^{\prime}\right)$ & $\begin{array}{c}\text { Product } \\
\text { length (bp) }\end{array}$ \\
\hline \multirow{2}{*}{$\beta$-actin } & F: CCTAGGCACCAGGGTGTGAT & \multirow{2}{*}{122} \\
\hline & R: TTGGTGACAATGCCGTGTTC & \\
\hline \multirow{2}{*}{ Akt2 } & F: CTCTGTAGCAGAATGCCAGC & \multirow{2}{*}{103} \\
\hline & R: ATGGAAGGTCCTCTCGATGA & \\
\hline \multirow{2}{*}{$\mathrm{aPKC} \zeta$} & F: CAGTGAAGGTGACCCTTGTA & \multirow{2}{*}{75} \\
\hline & R: GGACGAAGTGCTCATCATCC & \\
\hline \multirow{2}{*}{$\mathrm{aPKC} \lambda$} & F: GCTCACTCCAGATGATGATG & \multirow{2}{*}{120} \\
\hline & R: GGCAGTAAGCAGAATCAGAC & \\
\hline \multirow{2}{*}{ GLUT4 } & F: GTTGGTCTCGGTGCTCTTAG & \multirow{2}{*}{151} \\
\hline & R: GGCCACGATGGACACATAAC & \\
\hline \multirow{2}{*}{ PI3K-p85 $\alpha$} & F: ATACTTGATGTGGCTGACGC & \multirow{2}{*}{110} \\
\hline & R: AATCCTCGTCATCGTCTACC & \\
\hline \multirow{2}{*}{ IRS1 } & F: CTGGACGTCACAGGCAGAAT & \multirow{2}{*}{110} \\
\hline & R: CGTGAGGTCCTGGTTGTGAA & \\
\hline \multirow{2}{*}{ IRS2 } & F: GCCACCGTGGTGAAAGAGTA & \multirow{2}{*}{183} \\
\hline & R: CCTGCCTCTTGGTTCCTTAT & \\
\hline
\end{tabular}

F: forward; R: reverse.

IRS1: insulin receptor substrate 1; IRS2: insulin receptor substrate 2; PI3K: phosphatidylinositol-3 kinase; PKC: protein kinase C; GLUT4: glucose transporter 4; Akt2: protein kinase B beta.

levels. After blood sampling, rats were anesthetized using pentobarbital sodium ( $40 \mathrm{mg} / \mathrm{kg}$, intraperitoneal injection). Perfusions were carried out in the presence of $500 \mathrm{IU} / \mathrm{mL}$ insulin into the abdominal aorta for all experimental groups; this included a $5 \mathrm{~min}$ stabilization period and subsequent perfusion [27]. After perfusions, rats were sacrificed by dislocation, and the musculus quadriceps femoris was rapidly removed and stored at $-80^{\circ} \mathrm{C}$ until real-time RT-PCR and Western blot analysis were performed.

2.4. Plasma and Serum Analysis. FPG was measured by the glucose oxidase method. FINS and C-P were determined by enzyme linked immunosorbent assay (ELISA) (kits obtained from R\&D Co., USA). The ELISA method was performed according to the manufacturer's instructions. Additionally, the following formula was used to calculate homeostasis model assessment-estimated insulin resistance (HOMA-IR) $=$ FINs $(\mathrm{IU} / \mathrm{L}) \times$ FPG $(\mathrm{mmol} / \mathrm{L}) / 22.5$. This method has been previously described [28].

2.5. Quantitative Real-Time RT-PCR. Total RNA was isolated from musculus quadriceps femoris tissue using TRIzol (Invitrogen, Carlsbad, CA, USA). cDNA synthesis was performed using the MMLV Reverse Transcriptase 1st-Strand cDNA Synthesis Kit (TaKaRa, Dalian, China), according to the directions provided by the manufacturer. Specific mRNA quantification was performed by real-time PCR using SYBR ${ }^{\circledR}$ Green real-time PCR kit (TaKaRa) in a Real-Time PCR Detection System (B\&R). The gene-specific primers used for IRS1, IRS2, PI3Kp-85 $\alpha$, Akt2, aPKC $\zeta$, APKC $\lambda$, and GLUT4 are shown in Table 1. All reactions involved initial denaturation at $95^{\circ} \mathrm{C}$ for $2 \mathrm{~min}$ followed by 40 cycles of $95^{\circ} \mathrm{C}$ for $30 \mathrm{~s}$ and $60^{\circ} \mathrm{C}$ for $35 \mathrm{~s}$. The $2^{-\Delta \mathrm{Ct}}$ method was performed to calculate relative mRNA expression of target genes. $\beta$-actin was used as an internal control.

2.6. Western Blot. Western blot was performed to measure protein expression of PI3Kp85, pPKC $\zeta / \lambda$, and GLUT4 in muscle tissue samples. One milliliter of lysis buffer was added per milligram of protein. The tissue sample plus lysis buffer was then homogenized by cryogenic grinding. Pyrolysis was performed at $4^{\circ} \mathrm{C}$ for 3 hours. The supernatant was collected after centrifugation. For Western blot, equal amounts of protein $(30 \mu \mathrm{g})$ were separated by $10 \%$ SDSPAGE and electrophoretically transferred to nitrocellulose membrane. Nonspecific binding sites were blocked with 5\% milk powder diluted in TBS with $0.05 \%$ Tween 20 (TBST) for $60 \mathrm{~min}$. Proteins were detected using the following antibodies: rabbit polyclonal antibody for PI3K-p85 (diluted 1:3000; Bios; bs-0128R), rabbit polyclonal antibody for phosphoPKC $\zeta / \lambda$ (diluted 1:3000; CST; \#9378), rabbit polyclonal antibody for GLUT4 (diluted 1:3000; Boster; BA1626), and mouse monoclonal antibody for GAPDH (diluted 1:3000; Boster; BM1623). GAPDH was used as an internal control. Following overnight incubation with the primary antibody at $4^{\circ} \mathrm{C}$, each blot was washed three times with TBST buffer. Blots were then incubated with horseradish peroxidaseconjugated secondary antibodies (diluted 1:6000; Boster) at room temperature for $60 \mathrm{~min}$. Proteins were detected using an enhanced chemiluminescence reagent. Band intensity was quantified using SensiAnsys gel image analysis software.

2.7. Statistical Analysis. Statistical analysis was performed using SPSS analytical software 20.0 (SPSS Inc., Chicago, IL, USA). Data are expressed as mean \pm standard deviation $(x \pm s)$. Statistical significance was evaluated by one-way analysis of variance (ANOVA) with Student-Newman-Keuls (SNK) test for post hoc analysis. A $P$ value less than 0.05 was considered statistically significant.

\section{Results}

3.1. Effects of Acupuncture on Plasma FPG, Plasma FINS, Serum C-P, and HOMA-IR in OLETF Rats. Compared to the SD control group, FPG levels and HOMA-IR were significantly elevated in OLETF rats (both $P<0.05$ ). Additionally, FINS and C-P were also increased in the OLETF group compared to SD controls (both $P<0.01$ ) (Table 2). When compared to the SD group, levels of FPG and HOMA-IR were significantly decreased in the acupuncture+OLETF group (both $P<0.01$ ); in contrast, no significant differences in either FINS or C-P were detected between these two groups (both $P>0.05$ ). Levels of FPG, FINS, HOMA-IR, and C-P in the acupuncture+OLETF group were all significantly less than levels in the OLETF group (all $P<0.01$ ).

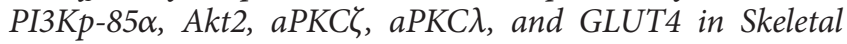


TABLE 2: Effects of acupuncture on plasma FPG, plasma FINS, serum C-P, and HOMA-IR in OLETF rats.

\begin{tabular}{|c|c|c|c|c|}
\hline Group & $\mathrm{FPG}\left(\mathrm{mmol} \cdot \mathrm{L}^{-1}\right)$ & FINS $\left(\mathrm{mU} \cdot \mathrm{L}^{-1}\right)$ & HOMA-IR & $C-P\left(n g \cdot L^{-1}\right)$ \\
\hline SD & $9.26 \pm 1.69$ & $56.30 \pm 0.74$ & $1.36 \pm 0.81$ & $277.84 \pm 10.96$ \\
\hline OLETF & $11.53 \pm 2.43^{*}$ & $59.53 \pm 2.02^{* *}$ & $1.48 \pm 0.86^{*}$ & $305.41 \pm 7.83^{* *}$ \\
\hline Acupuncture+OLETF & $6.19 \pm 0.93^{* * \Delta \Delta}$ & $56.67 \pm 1.80^{\triangle \triangle}$ & $1.19 \pm 0.66^{* * \Delta \Delta}$ & $286.44 \pm 10.82^{\triangle \Delta}$ \\
\hline
\end{tabular}

Data are shown as mean \pm standard deviation (SD) ( $n=8$ each group).

${ }^{*} P<0.05$ and ${ }^{* *} P<0.01$ versus SD group; ${ }^{\triangle} P<0.01$ versus OLETF group.

FPG: fasting blood glucose; FINS: fasting insulin; C-P: C peptide; HOMA-IR: homeostasis model assessment-estimated insulin resistance; OLETF: Otsuka Long-Evans Tokushima Fatty; SD: Sprague-Dawley.

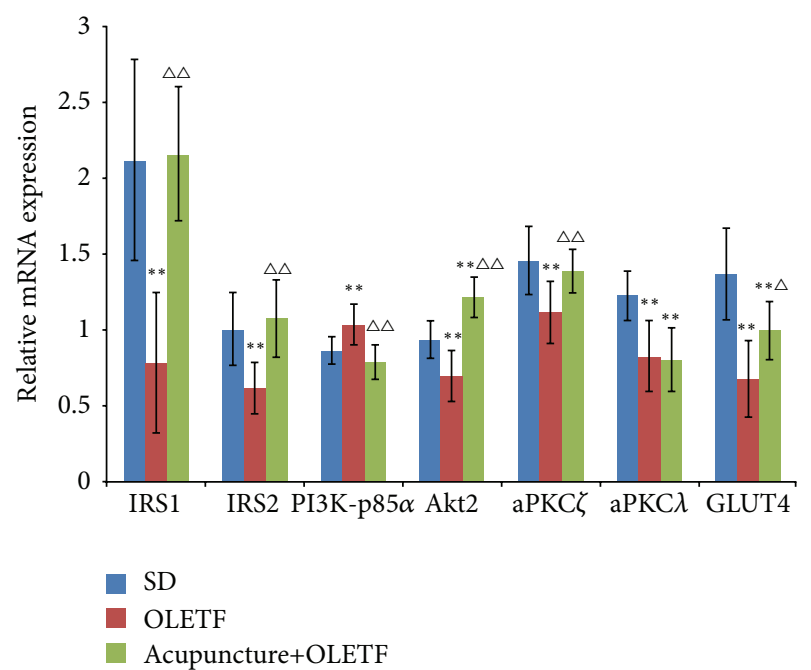

FIGURE 2: Effects of acupuncture on mRNA expressions of IRS1, IRS2, PI3K-p85 $\alpha$, Akt2, aPKC $\zeta$, aPKC $\lambda$, and GLUT4 in skeletal muscle of OLETF rats. Acupuncture was applied to the indicated region for $20 \mathrm{~min}$ per day, over the course of 21 days. Following administration, the musculus quadriceps femoris was obtained. Relative mRNA expression was determined by real-time RT-PCR. Data are showed as mean \pm standard deviation (SD) $(n=8$ each group). ${ }^{* *} P<0.01$ versus SD group; ${ }^{\triangle} P<0.05$ and ${ }^{\triangle}{ }^{\triangle} P<$ 0.01 versus OLETF group. IRS1: insulin receptor substrate 1; IRS2: insulin receptor substrate 2; PI3K: phosphatidylinositol-3 kinase; PKC: protein kinase C; GLUT4: glucose transporter 4; Akt2: protein kinase B beta.

Muscle of OLETF Rats. mRNA expression of IRS1, IRS2, Akt2, aPKC $\zeta$, aPKC $\lambda$, and GLUT4 were all significantly reduced in skeletal muscle from OLETF rats compared to skeletal muscle from control SD rats (Figure 2) (all $P<0.01$ ). In contrast, mRNA expression of PI3K-p85 $\alpha$ was significantly higher in OLETF rats compared to controls $(P<0.01)$. mRNA expression of Akt2 was significantly higher in the acupuncture+OLETF group compared to the SD control group $(P<0.01)$. In contrast, mRNA expressions of GLUT4 and aPKC $\lambda$ were reduced in the acupuncture+OLETF group compared to SD controls (both $P<0.01$ ); expressions of other genes IRS1, IRS2, PI3K-p85 $\alpha$, and aPKC $\zeta$ were not significantly altered between these two groups (all $P>0.05$ ). When comparing the acupuncture+OLETF group to the OLETF group alone, we find that mRNA expressions of IRS-1, IRS-2, Akt2, aPKC $\zeta$, and GLUT4 are all significantly elevated in the animals receiving acupuncture treatment (all $P<0.05$ ); in contrast, transcript level of PI3K-p $85 \alpha$ was significantly reduced $(P<0.01)$ upon acupuncture.

3.3. Effects of Acupuncture on Protein Expression of PI3Kp85, pPKC $\zeta / \lambda$, and GLUT4 in Skeletal Muscle of OLETF Rats. Protein expression of PI3K-p85 was significantly increased $(P<0.01)$ in OLETF rats compared to SD controls (Figure 3$)$; importantly, this increase was attenuated by acupuncture treatment. In contrast, GLUT4 protein levels were reduced in the OLETF group compared to the control SD group $(P<0.01)$; again, we find that acupuncture attenuated this decrease in GLUT4 observed in the OLETF animals. Unlike PI3K-p85 and GLUT4, protein expression of phospho$\mathrm{PKC} \zeta / \lambda$ in the acupuncture+OLETF group was significantly increased compared to SD controls $(P<0.01)$, but not significantly altered compared to OLETF rats $(P>0.05)$.

\section{Discussion}

Acupuncture has been used as a remedy for a number of malignancies, including as a method of treatment to improve whole body metabolism. Several studies have found that acupuncture improves whole body glucose tolerance in human subjects of various ages and genders [4, 13]. It has been suggested that the improvements in whole body glucose tolerance are a result of increased body sensitivity that enhances glucose disposal from the body. Many of these same studies also showed that acupuncture could effectively reduce blood glucose concentration. However, it is plausible that acupuncture may also facilitate qualitative changes in skeletal muscle which contribute to the improvements in glucose metabolism. In the present investigation, we used genetically hyperglycemic rats as a model to evaluate the influence of acupuncture on skeletal muscle glucose uptake and transport. Furthermore, inclusion of the SD control group allowed us to assess specific adaptations in skeletal muscle glucose metabolism which occur in response to acupuncture. The results from this study show that acupuncture improves insulin resistance in OLETF rats, possibly via regulating expression of key insulin signaling related molecules.

Following acupuncture administration, we determined levels of plasma FPG, plasma FINS, HOMA-IR, and serum $\mathrm{C}-\mathrm{P}$ as a measure of treatment efficacy. We also examined the influence of acupuncture treatment on expression levels of key insulin signaling components in skeletal muscle. OLETF rats exhibit significant insulin resistance with elevated levels 


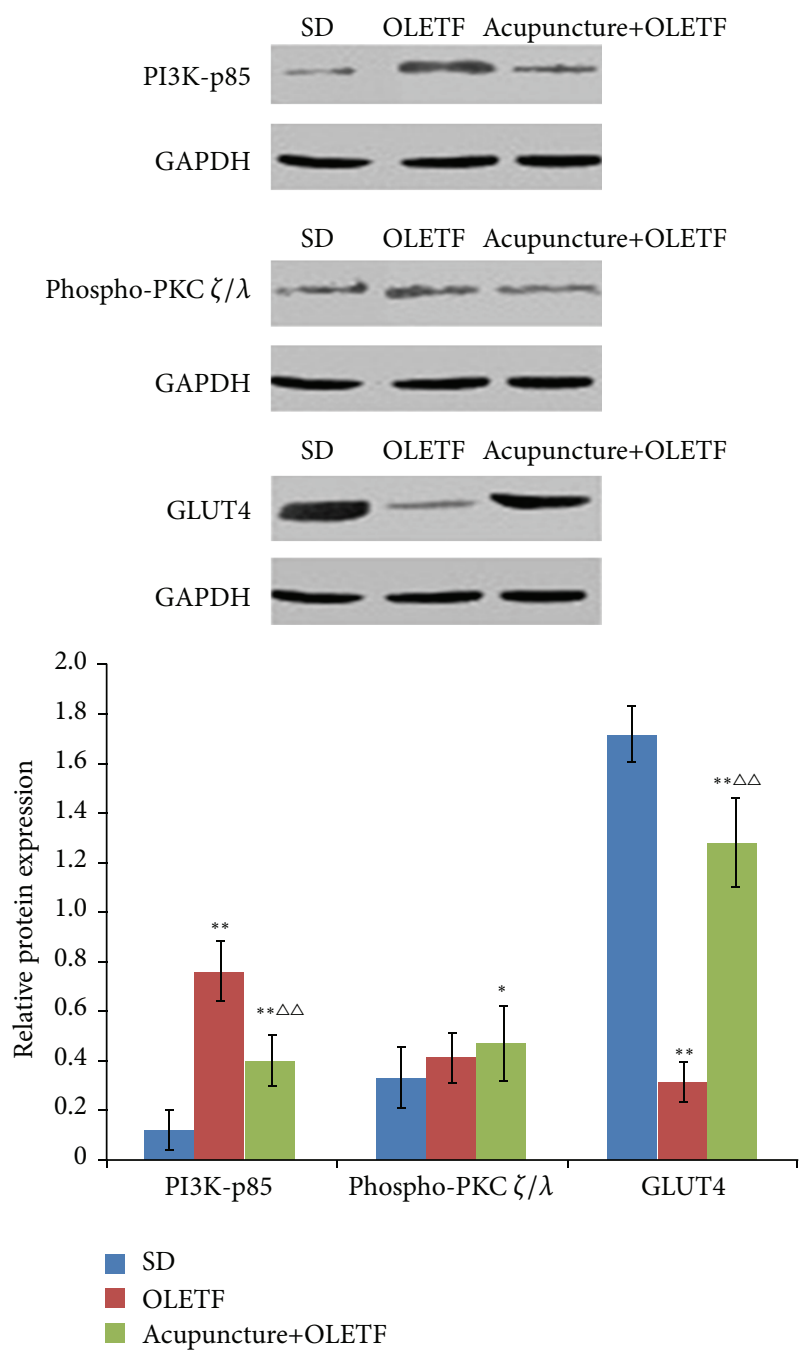

FIgURE 3: Effects of acupuncture on protein expressions of PI3Kp85, phospho-PKC $\zeta / \lambda$, and GLUT4 in skeletal muscle of OLETF rats. Protein expression was determined by Western blot. GAPDH was used as an internal control. Data are shown as mean $\pm \mathrm{SD}(n=8$ each group). ${ }^{*} P<0.05$ and ${ }^{* *} P<0.01$ versus SD group; ${ }^{\triangle \triangle} P<0.01$ versus OLETF group.

of FPG, FINS, HOMA-IR, and C-P. Importantly, we find that many of these parameters are decreased to normal levels following acupuncture treatment. These findings raise the interesting possibility that improved insulin sensitivity following acupuncture administration may be due to alterations in components of the insulin signaling cascade. Along these lines, we find increased expression of IRS-1/2 after acupuncture, which likely contributes to improved rates of insulin-stimulated glucose uptake and transport. This is consistent with other reports showing that altered expression or activity of insulin-related molecules, for example, PI 3kinase, is associated with altered rates of insulin-stimulated glucose uptake and transport $[29,30]$. Also consistent with other reports, we find that 21 days of acupuncture administration increases PI 3-kinase activity in normal rodent skeletal muscle. Thus, acupuncture improves symptoms associated with insulin resistance possibly by altering the expression or activity of PI3K.

We next examined the effect of acupuncture on proteins downstream of PI3K in the insulin signal transduction cascade. Several earlier studies have shown that Akt phosphorylation is impaired in insulin-resistant tissue [31, 32]; this is in contrast to other reports stating that Akt phosphorylation is unaffected by insulin resistance $[33,34]$. There is clearly some question surrounding Akt phosphorylation in response to insulin resistance, and there may very well be a tissue-type specific effect. To support this possibility, one study reported that chronic aerobic exercise increased insulin-stimulated Akt2 activity, but only within the red gastrocnemius in normal rodent skeletal muscle [35]. Importantly, it is Akt2 that accounts for the majority of insulin-regulated glucose uptake [36]. As such, these data clearly implicate Akt2 as the key isoform involved in this process and suggest that future efforts be directed toward understanding how activation of Akt2 is regulated. In this study, we observed decreased expression of Akt 2 in OLETF rats compared to controls. We also show that acupuncture administration significantly attenuates the OLETF-mediated decrease in Akt2. Therefore, our findings suggest that insulin resistance in the OLETF model is associated with decreased Akt2 expression and that acupuncture may relieve some of the insulin-resistance related symptoms by restoring Akt2 expression back to normal levels.

$\mathrm{PKC}-\zeta / \lambda$ is another downstream target of PI 3-kinase, which is suppressed in insulin-resistant skeletal muscle [31]; $\mathrm{PKC}-\zeta / \lambda$ also appears to be negatively affected by high-fat feeding [37]. As such, we examined expression and activity of PKC- $\zeta / \lambda$ in skeletal muscle of OLETF rats following acupuncture treatment. Expression of PKC- $\zeta / \lambda$ at the mRNA level was minimally altered in the OLETF model or by acupuncture administration. Importantly, activation of $\mathrm{PKC}-\zeta / \lambda$, assessed by phosphospecific antibody staining by Western blot, was unaltered in the OLETF model with or without acupuncture. Thus, we conclude that signaling via $\mathrm{PKC}-\zeta / \lambda$ is not a major contributing factor mediating glucose metabolism in skeletal muscle of OLETF rats.

While several studies have evaluated the effects of insulinstimulated IRS-1/2-associated PI 3-kinase activity on glucose metabolism, it has recently been demonstrated that activation of PI 3-kinase alone may not be sufficient to fully account for insulin-stimulated glucose transport [38]. While insulin signaling has garnered some preliminary evaluation in skeletal muscle from genetically [39] and pharmacologically induced models [40] of insulin resistance, to the best of our knowledge, the present investigation is the first to assess insulin signaling activation in a normal rodent model of diabetes that were subjected to aerobic exercise. The specific role of insulin signaling in the regulation of insulin-stimulated glucose transport is unclear; however, the translocation of GLUT4 may be involved [41-43]. Consistent with this possibility, our findings show that acupuncture enhances expression of GLUT4 at both the protein and mRNA levels. This acupuncture-mediated increase may promote glucose transport and help alleviate some of the symptoms associated with insulin resistance. 
The work we present here suggests that acupuncture improves insulin resistance in OLETF rats, possibly via regulating expression of key insulin signaling related molecules. However, we only managed to detect the expression of these proteins, among which some remained unchanged in their expression or activity during the experiment. As such, this represents an important limitation of our study. Additional work should be performed in which the effect of acupuncture on insulin resistance is determined in the presence of PI3K/Akt pathway inhibition, maybe, for example, with the use of small molecule inhibitors.

\section{Conclusions}

In summary, rodents subjected to 21 days of acupuncture display improved insulin sensitivity, which cooccurs with changes in expression of several components of the insulin signaling pathway, such as PI3K, Akt, and GLUT4. Thus, acupuncture may represent a useful therapeutic option for patients with T2DM.

\section{Competing Interests}

The authors declare that they have no competing interests.

\section{Authors' Contributions}

Liang Zhang and Jian Sun contributed equally to this work.

\section{Acknowledgments}

The authors thank Dr. Neng-Gui Xu and Dr. Wei Yi for their guidance on this work. The authors also thank Dr. Liang Zhang for technical advice and Dr. Jian Sun for excellent technical assistance. This investigation was supported by the National Natural Science Foundation of China (no. 30873294, no. 81273845 , no. 81303043 , and no. 81303042), the Guangdong Natural Science Foundation (no. 9351040701000001), and the Science and Technology Planning Project of Guangdong Province, China (no. 2010B030700033).

\section{References}

[1] C. V. Rizos, M. S. Elisaf, D. P. Mikhailidis, and E. N. Liberopoulos, "How safe is the use of thiazolidinediones in clinical practice?” Expert Opinion on Drug Safety, vol. 8, no. 1, pp. 15-32, 2009.

[2] O.-P. R. Hamnvik and G. T. McMahon, "Balancing risk and benefit with oral hypoglycemic drugs," Mount Sinai Journal of Medicine, vol. 76, no. 3, pp. 234-243, 2009.

[3] N. Ishizaki, N. Okushi, T. Yano, and Y. Yamamura, "Improvement in glucose tolerance as a result of enhanced insulin sensitivity during electroacupuncture in spontaneously diabetic Goto-Kakizaki rats," Metabolism: Clinical and Experimental, vol. 58, no. 10, pp. 1372-1378, 2009.

[4] S. L. Chang, J. G. Lin, T. C. Chi, I. M. Liu, and J. T. Cheng, "An insulin-dependent hypoglycaemia induced by electroacupuncture at the Zhongwan (CV12) acupoint in diabetic rats," Diabetologia, vol. 42, no. 2, pp. 250-255, 1999.
[5] M. Y. Shapira, E. Y. Appelbaum, B. Hirshberg, Y. Mizrahi, H. Bar-On, and E. Ziv, "A sustained, non-insulin related, hypoglycaemic effect of electroacupuncture in diabetic Psammomys obesus," Diabetologia, vol. 43, no. 6, pp. 809-813, 2000.

[6] K. W. Chen, T. Liu, H. Zhang, and Z. Lin, "An analytical review of the Chinese literature on Qigong therapy for diabetes mellitus," The American Journal of Chinese Medicine, vol. 37, no. 3, pp. 439-457, 2009.

[7] R. Taylor, "Aetiology of non-insulin dependent diabetes," British Medical Bulletin, vol. 45, no. 1, pp. 73-91, 1989.

[8] Y.-J. Li and H.-X. Xu, "Research progress on anti-diabetic Chinese medicines," Zhong Yao Cai, vol. 29, no. 6, pp. 621-624, 2006.

[9] A. M. Castro-Sánchez, C. Moreno-Lorenzo, G. A. MataránPeñarrocha, B. Feriche-Fernández-Castanys, G. GranadosGámez, and J. M. Quesada-Rubio, "Connective tissue reflex massage for type 2 diabetic patients with peripheral arterial disease: randomized controlled trial," Evidence-Based Complementary and Alternative Medicine, vol. 2011, Article ID 804321, 12 pages, 2011.

[10] M. Chao, D. Zou, Y. Zhang et al., "Improving insulin resistance with traditional Chinese medicine in type 2 diabetic patients," Endocrine, vol. 36, no. 2, pp. 268-274, 2009.

[11] Y. Fukazawa, T. Maeda, and S. Kishioka, "The pharmacological mechanisms of electroacupuncture," Current Opinion in Investigational Drugs, vol. 10, no. 1, pp. 62-69, 2009.

[12] F. J. Zijlstra, I. van den Berg-de Lange, F. J. P. M. Huygen, and J. Klein, "Anti-inflammatory actions of acupuncture," Mediators of Inflammation, vol. 12, no. 2, pp. 59-69, 2003.

[13] S.-L. Chang, K.-J. Lin, R.-T. Lin, P.-H. Hung, J.-G. Lin, and J.-T. Cheng, "Enhanced insulin sensitivity using electroacupuncture on bilateral Zusanli acupoints (ST 36) in rats," Life Sciences, vol. 79, no. 10, pp. 967-971, 2006.

[14] F. Liang and D. Koya, "Acupuncture: is it effective for treatment of insulin resistance?" Diabetes, Obesity and Metabolism, vol. 12, no. 7, pp. 555-569, 2010.

[15] N.-N. Chu, W. Xia, P. Yu, L. Hu, R. Zhang, and C.-L. Cui, "Chronic morphine-induced neuronal morphological changes in the ventral tegmental area in rats are reversed by electroacupuncture treatment," Addiction Biology, vol. 13, no. 1, pp. $47-51,2008$.

[16] A. Danielsson, A. Öst, F. H. Nystrom, and P. Strålfors, "Attenuation of insulin-stimulated insulin receptor substrate-1 serine 307 phosphorylation in insulin resistance of type 2 diabetes," The Journal of Biological Chemistry, vol. 280, no. 41, pp. 3438934392, 2005.

[17] J. Jager, T. Grémeaux, M. Cormont, Y. Le Marchand-Brustel, and J.-F. Tanti, "Interleukin- $\beta$-induced insulin resistance in adipocytes through down-regulation of insulin receptor substrate-1 expression," Endocrinology, vol. 148, no. 1, pp. 241251, 2007.

[18] A. Herschkovitz, Y.-F. Liu, E. Ilan, D. Ronen, S. Boura-Halfon, and Y. Zick, "Common inhibitory serine sites phosphorylated by IRS-1 kinases, triggered by insulin and inducers of insulin resistance," The Journal of Biological Chemistry, vol. 282, no. 25, pp. 18018-18027, 2007.

[19] T. Kadowaki, "Insights into insulin resistance and type 2 diabetes from knockout mouse models," The Journal of Clinical Investigation, vol. 106, no. 4, pp. 459-465, 2000.

[20] G. Yuan, J. Deng, T. Wang et al., “Tissue kallikrein reverses insulin resistance and attenuates nephropathy in diabetic rats 
by activation of phosphatidylinositol 3-kinase/protein kinase $\mathrm{B}$ and adenosine $5^{\prime}$-monophosphate-activated protein kinase signaling pathways," Endocrinology, vol. 148, no. 5, pp. 20162026, 2007.

[21] M. J. Charron, N. Gorovits, J. S. Laidlaw, M. Ranalletta, and E. B. Katz, "Use of GLUT-4 null mice to study skeletal muscle glucose uptake," Clinical and Experimental Pharmacology and Physiology, vol. 32, no. 4, pp. 308-313, 2005.

[22] H. Alkhateeb, A. Chabowski, J. F. C. Glatz, J. F. P. Luiken, and A. Bonen, "Two phases of palmitate-induced insulin resistance in skeletal muscle: impaired GLUT4 translocation is followed by a reduced GLUT4 intrinsic activity," American Journal of Physiology_Endocrinology and Metabolism, vol. 293, no. 3, pp. E783-E793, 2007.

[23] K. Kawano, T. Hirashima, S. Mori, Y. Saitoh, M. Kurosumi, and T. Natori, "Spontaneous long-term hyperglycemic rat with diabetic complications. Otsuka Long-Evans Tokushima Fatty (OLETF) strain," Diabetes, vol. 41, no. 11, pp. 1422-1428, 1992.

[24] Y. Mori, J. Yokoyama, M. Nemoto, S. Katoh, M. Nishimura, and Y. Ikeda, "Characterization of a new genetically obesehyperglycemic OLETF rat," The Jikeikai Medical Journal, vol. 39, pp. 349-359, 1992.

[25] K. Kawano, T. Hirashima, S. Mori, and T. Natori, "OlETF (Otsuka Long-Evans Tokushima Fatty) rat: a new NIDDM rat strain," Diabetes Research and Clinical Practice, vol. 24, supplement, pp. S317-S320, 1994.

[26] W. Z. Lin and P. Wang, Experimental Acupuncture, Shanghai Scientific \& Technical Publisher, Shanghai, China, 1994.

[27] B. B. Yaspelkis III, M. K. Singh, B. Trevino, A. D. Krisan, and D. E. Collins, "Resistance training increases glucose uptake and transport in rat skeletal muscle," Acta Physiologica Scandinavica, vol. 175, no. 4, pp. 315-323, 2002.

[28] D. R. Matthews, J. P. Hosker, A. S. Rudenski, B. A. Naylor, D. F. Treacher, and R. C. Turner, "Homeostasis model assessment: insulin resistance and $\beta$-cell function from fasting plasma glucose and insulin concentrations in man," Diabetologia, vol. 28, no. 7, pp. 412-419, 1985.

[29] M. K. Singh, A. D. Krisan, A. M. Crain, D. E. Collins, and B. B. Yaspelkis III, "High-fat diet and leptin treatment alter skeletal muscle insulin-stimulated phosphatidylinositol 3kinase activity and glucose transport," Metabolism: Clinical and Experimental, vol. 52, no. 9, pp. 1196-1205, 2003.

[30] A. D. Krisan, D. E. Collins, A. M. Crain et al., "Resistance training enhances components of the insulin signaling cascade in normal and high-fat-fed rodent skeletal muscle," Journal of Applied Physiology, vol. 96, no. 5, pp. 1691-1700, 2004.

[31] A. Oku, M. Nawano, K. Ueta et al., "Inhibitory effect of hyperglycemia on insulin-induced Akt/protein kinase B activation in skeletal muscle," American Journal of PhysiologyEndocrinology and Metabolism, vol. 280, no. 5, pp. E816-E824, 2001.

[32] P. Vollenweider, B. Menard, and P. Nicod, "Insulin resistance, defective insulin receptor substrate 2-associated phosphatidylinositol-3' kinase activation, and impaired atypical protein kinase $\mathrm{C}$ (zeta/lambda) activation in myotubes from obese patients with impaired glucose tolerance," Diabetes, vol. 51, no. 4, pp. 1052-1059, 2002.

[33] Y.-B. Kim, S. E. Nikoulina, T. P. Ciaraldi, R. R. Henry, and B. B. Kahn, "Normal insulin-dependent activation of Akt/protein kinase B, with diminished activation of phosphoinositide 3kinase, in muscle in type 2 diabetes," The Journal of Clinical Investigation, vol. 104, no. 6, pp. 733-741, 1999.
[34] Y. T. Kruszynska, D. S. Worrall, J. Ofrecio, J. P. Frias, G. Macaraeg, and J. M. Olefsky, "Fatty acid-induced insulin resistance: decreased muscle PI3K activation but unchanged Akt phosphorylation," The Journal of Clinical Endocrinology o Metabolism, vol. 87, no. 1, pp. 226-234, 2002.

[35] J. R. Bernard, A. M. Crain, D. A. Rivas, H. J. Herr, D. W. Reeder, and B. B. Yaspelkis III, "Chronic aerobic exercise enhances components of the classical and novel insulin signalling cascades in Sprague-Dawley rat skeletal muscle," Acta Physiologica Scandinavica, vol. 183, no. 4, pp. 357-366, 2005.

[36] Z. Y. Jiang, Q. L. Zhou, K. A. Coleman, M. Chouinard, Q. Boese, and M. P. Czech, "Insulin signaling through Akt/protein kinase B analyzed by small interfering RNA-mediated gene silencing," Proceedings of the National Academy of Sciences of the United States of America, vol. 100, no. 13, pp. 7569-7574, 2003.

[37] F. Tremblay, C. Lavigne, H. Jacques, and A. Marette, "Defective insulin-induced GLUT4 translocation in skeletal muscle of high fat-fed rats is associated with alterations in both Akt/protein kinase B and atypical protein kinase $\mathrm{C}(\zeta / \lambda)$ activities," Diabetes, vol. 50, no. 8, pp. 1901-1910, 2001.

[38] T. Jiang, G. Sweeney, M. T. Rudolf, A. Klip, A. Traynor-Kaplan, and R. Y. Tsien, "Membrane-permeant esters of phosphatidylinositol 3,4,5-trisphosphate," The Journal of Biological Chemistry, vol. 273, no. 18, pp. 11017-11024, 1998.

[39] G. D. Wadley, C. R. Bruce, N. Konstantopoulos et al., "The effect of insulin and exercise on c-Cbl protein abundance and phosphorylation in insulin-resistant skeletal muscle in lean and obese Zucker rats," Diabetologia, vol. 47, no. 3, pp. 412-419, 2004.

[40] A. C. P. Thirone, J. B. C. Carvalheira, A. E. Hirata, L. A. Velloso, and M. J. A. Saad, "Regulation of Cbl-associated protein/Cbl pathway in muscle and adipose tissues of two animal models of insulin resistance," Endocrinology, vol. 145, no. 1, pp. 281-293, 2004.

[41] C. A. Baumann, V. Ribon, M. Kanzaki et al., "CAP defines a second signalling pathway required for insulin-stimulated glucose transport," Nature, vol. 407, no. 6801, pp. 202-207, 2000.

[42] S.-H. Chiang, C. A. Baumann, M. Kanzaki et al., "Insulinstimulated GLUT4 translocation requires the CAP-dependent activation of TC10," Nature, vol. 410, no. 6831, pp. 944-948, 2001.

[43] J. Liu, S. M. DeYoung, J. B. Hwang, E. E. O’Leary, and A. R. Saltiel, "The roles of Cbl-b and c-Cbl in insulin-stimulated glucose transport," The Journal of Biological Chemistry, vol. 278, no. 38, pp. 36754-36762, 2003. 


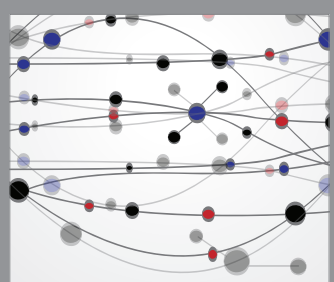

The Scientific World Journal
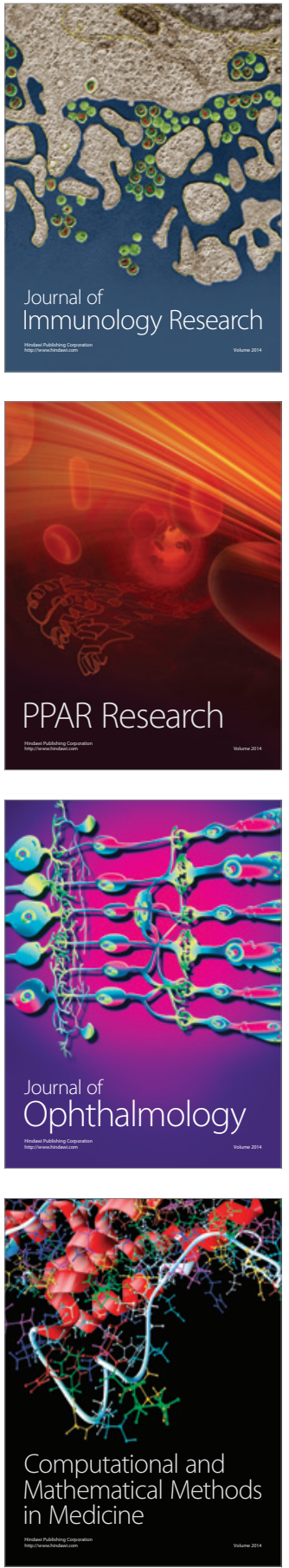

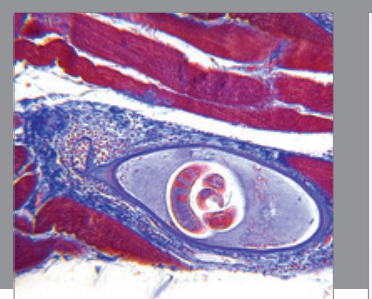

Gastroenterology Research and Practice

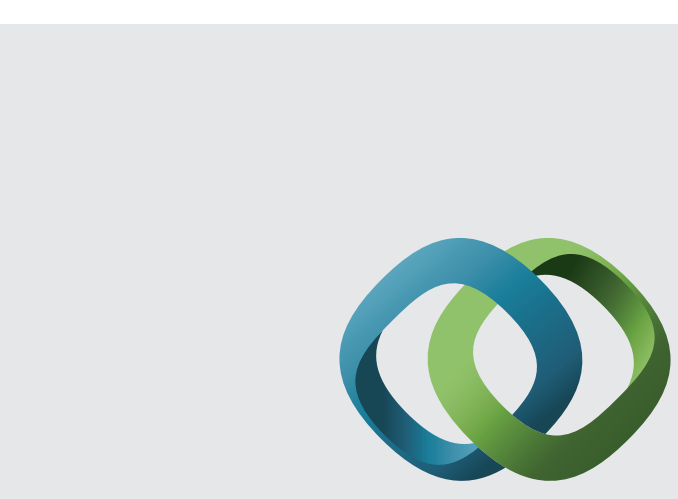

\section{Hindawi}

Submit your manuscripts at

http://www.hindawi.com
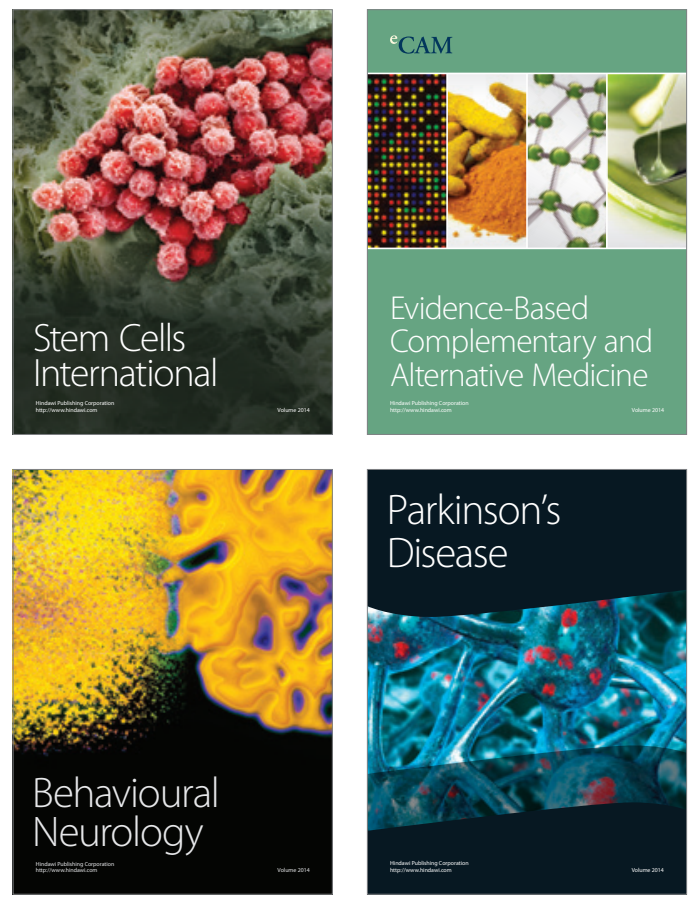
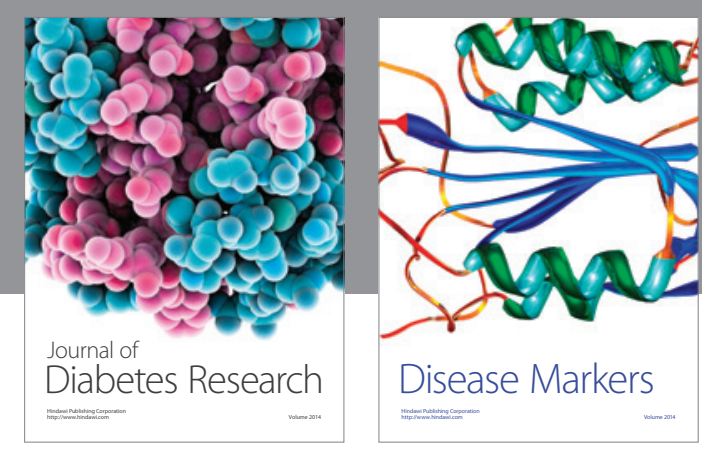

Disease Markers
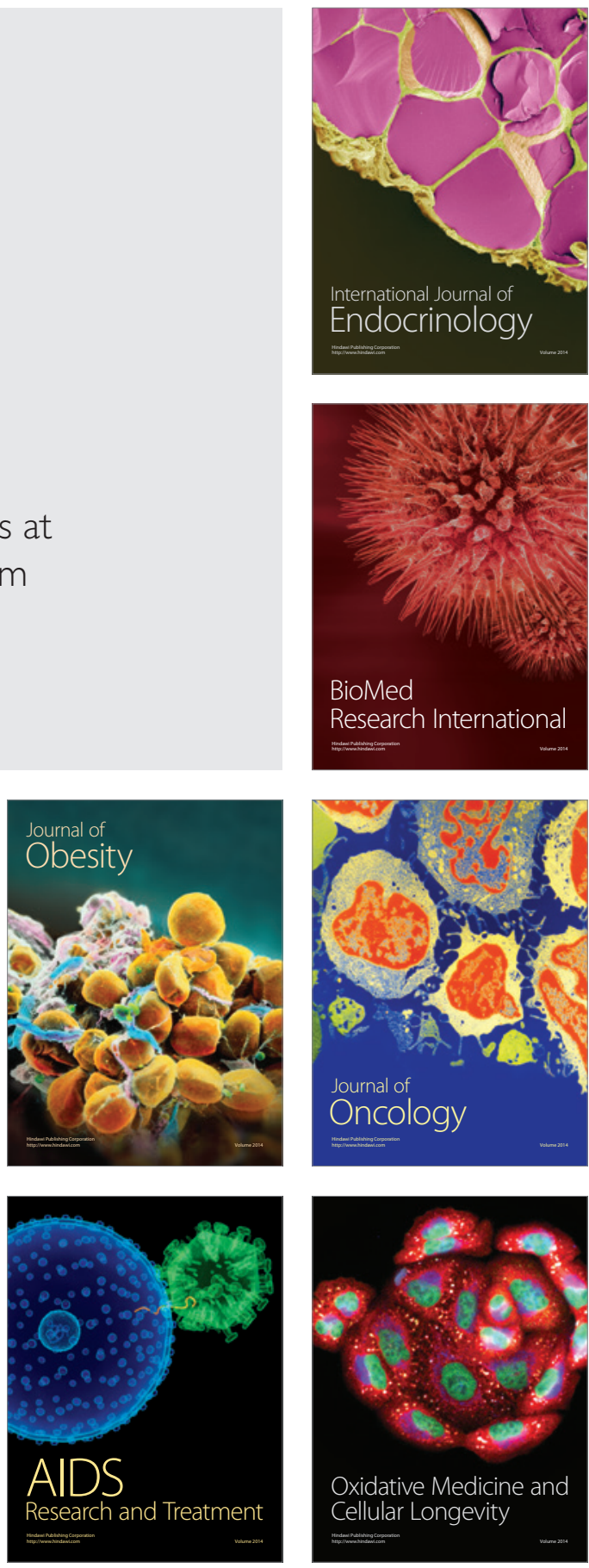